\title{
VIGILÂNCIA EM SAÚDE MENTAL NO CORPO DE BOMBEIROS MILITAR DE MINAS GERAIS (CBMMG)
}

\author{
Eduardo de Paula Lima' \\ Alina Gomide Vasconcelos ${ }^{2}$ \\ Bruno Henriques de Camargos ${ }^{3}$
}

\section{RESUMO}

A exposição de bombeiros militares a estressores ocupacionais aumenta os riscos à saúde mental. Ações de Vigilância em Saúde do Trabalhador (Visat) são recomendadas para a prevenção de doenças e promoção da saúde. $O$ objetivo do presente artigo foi descrever dois programas de Visat no Corpo de Bombeiros Militar de Minas Gerais (CBMMG): o Programa de Saúde Ocupacional Bombeiro Militar e o Estudo da Saúde do Bombeiro. Ambos focalizam estressores ocupacionais e desfechos em saúde mental. Buscam também oferecer subsídios para intervenções individuais e organizacionais. Benefícios alcançados são elencados e desafios para o avanço das ações de Visat no CBMMG são discutidos. A descrição dos programas em andamento em Minas Gerais pretende contribuir para o debate sobre saúde mental de bombeiros militares no Brasil.

Palavras-chave: Vigilância em Saúde; Saúde do Trabalhador; Saúde Mental; Bombeiros.

\footnotetext{
${ }^{1}$ Corpo de Bombeiros Militar de Minas Gerais. Universidade Federal de Minas Gerais. Psicólogo, Mestre em Psicologia, Doutor em Saúde Pública. Email: eduardo.lima@bombeiros.mg.gov.br ${ }^{2}$ Corpo de Bombeiros Militar de Minas Gerais. Universidade Federal de Minas Gerais. Psicóloga, Mestre em Psicologia, Doutora em Neurociência. Email: alina.vasconcelos@bombeiros.mg.gov.br ${ }^{3}$ Universidade Federal de Minas Gerais. Graduando em Psicologia. Email: brunohcamargos@hotmail.com
} 


\title{
OCCUPATIONAL MENTAL HEALTH SURVEILLANCE AT THE MINAS GERAIS FIRE DEPARTMENT
}

\begin{abstract}
Firefighters' exposure to occupational stressors increases risks of mental health problems. Occupational Health Surveillance (OHS) actions are recommended for disease prevention and health promotion. This paper aimed to describe two OHS programs developed at the Minas Gerais Fire Department: the Firefighter Occupational Health Program and the Firefighter Longitudinal Health Study. Both programs focus on occupational stressors and mental health outcomes. They also aim to provide subsidies for individual and organizational interventions. Benefits achieved are listed and challenges for developing OHS at Minas Gerais Fire Department are discussed. The description of programs in progress in Minas Gerais aims to contribute to the debate on the mental health of firefighters in Brazil.
\end{abstract}

Keywords: Health Surveillance; Occupational Health; Mental Health; Firefighters 


\section{INTRODUÇÃO}

A prevenção do adoecimento psíquico e a promoção da saúde mental são desafios atuais para a área da Saúde do Trabalhador. A prevalência de transtornos mentais tem crescido nos últimos anos, tanto no Brasil quanto em outros países ao redor do mundo (STEEL et al., 2014). Entre trabalhadores ativos, tal crescimento implica em comprometimento da qualidade de vida, perda de produtividade, afastamento temporário do trabalho ou aposentadoria precoce (GOETZEL et al., 2018). No âmbito previdenciário brasileiro, por exemplo, o mais recente Anuário Estatístico da Previdência Social indicou que transtornos mentais e do comportamento (diagnosticados de acordo com a Classificação Internacional de Doenças) têm ocupado a terceira posição na concessão de auxílio-doença por incapacidade. Apenas em 2017, foram registrados 162.548 casos (BRASIL, 2017).

Ao voltar a atenção para a realidade dos bombeiros militares, o cenário não é diferente daquele observado entre os trabalhadores brasileiros em geral. Estudo realizado no município de Campo Grande, Mato Grosso do Sul, mostrou que o absenteísmo por transtornos mentais e do comportamento entre bombeiros militares cresceu nos últimos anos, compondo uma das principais causas de afastamento do trabalho naquele estado (FIORIN, 2013). Em Santa Catarina, no período entre 2013 e 2016, os transtornos mentais foram a terceira causa de afastamento (PEREIRA, 2017). Inquéritos sobre saúde mental entre bombeiros militares brasileiros seguem na mesma direção: são registradas altas prevalências de transtornos mentais, incluindo transtorno de estresse pós-traumático (LIMA, BARRETO, ASSUNÇÃO, 2015; BERGER et al., 2007), uso nocivo de álcool (AMATO et al., 2010) e quadros de burnout (LOPES, 2010).

A magnitude do problema e suas consequências para os bombeiros militares sugerem que é necessária a adoção de ações institucionais visando a 
saúde mental. Dentre elas, é possível destacar as ações de Vigilância em Saúde do Trabalhador - Visat (LEÃO, GOMEZ, 2014). Tais ações podem utilizar dados já disponíveis (vigilância passiva) ou empreender esforços no sentido de construir ferramentas para identificar e registrar casos de adoecimento e acidentes de trabalho (vigilância ativa). As duas modalidades podem também ser combinadas, criando-se um sistema híbrido. Contudo, independente da modalidade adotada, alguns desafios estão sempre presentes, como registro de informações, construção de indicadores, análise e interpretação dos dados, retroalimentação (fase que requer a apresentação e discussão dos resultados junto aos trabalhadores e gestores da instituição) e planejamento e implementação de intervenções (MAIZLISH, 2000).

Leão e Gomez (2014) chamam a atenção ainda para um aspecto importante a ser observado nas ações em Visat: não basta focalizar a presença de sintomas psíquicos, é necessário avaliar e compreender sua relação com os fatores de risco presentes no ambiente de trabalho. Cabe enfatizar que, na realidade dos bombeiros militares, há dois grandes grupos de fatores de risco para a saúde mental: estressores operacionais e estressores organizacionais (LIMA, 2013).

O primeiro, estressores operacionais, está ligado à natureza das tarefas e decorre da exposição rotineira a situações de violência, risco de morte ou morte de pessoas, incluindo o risco para a vida dos próprios bombeiros (VAN DER VELDEN, KLEBER, GRIEVINK, YZERMANS, 2010). As cenas presenciadas pelos trabalhadores em ocorrências de atendimento préhospitalar, salvamento e combate a incêndio urbano são destaques nesse grupo (MONTEIRO, LIMA, 2018).

O segundo, estressores organizacionais, é comum aos trabalhadores em geral, embora assuma características peculiares nos Corpos de Bombeiros. Se vincula à forma pela qual o trabalho é organizado nas instituições. É possível citar ritmo de trabalho, pressão de tempo, número de horas trabalhadas, grau de autonomia sobre as tarefas, possibilidade de ter voz ativa 
nas decisões que interferem no trabalho de cada um e apoio social de colegas e chefes (KARASEK, THEORELL, 1990; GLINA, ROCHA, 2010). No conjunto, tanto estressores operacionais quanto organizacionais são essenciais para 0 entendimento da dinâmica saúde mental-trabalho (LIMA, ASSUNÇÃO, 2011).

Considerando as recomendações sobre Visat e suas metas institucionais de planejamento estratégico (MINAS GERAIS, 2017), o Corpo de Bombeiros Militar de Minas Gerais (CBMMG) desenvolveu uma ferramenta de vigilância ativa, o Programa de Saúde Ocupacional Bombeiro Militar - PSOBM (MINAS GERAIS, 2015). O objetivo do PSOBM é realizar avaliações periódicas dos bombeiros militares do estado, mensurando a exposição a fatores de risco presentes no ambiente de trabalho e suas possíveis consequências à saúde. 0 programa inclui a atenção à saúde física e mental, ou seja, tem um caráter multidisciplinar pouco usual no Brasil. Vale frisar: a saúde mental é um aspecto raramente focalizado em ações de Visat (LEÃO, GOMEZ, 2014).

De forma complementar ao PSOBM, a equipe de psicologia do CBMMG, em parceria com a Universidade Federal de Minas Gerais (UFMG), desenvolveu um projeto de acompanhamento periódico dos soldados que ingressaram na instituição a partir de 2014. Trata-se de um estudo longitudinal denominado Estudo da Saúde do Bombeiro - ESB (TEOH, LIMA, VASCONCELOS, NASCIMENTO, COX, 2019). O foco é mensurar características individuais e contextuais que podem proteger ou colocar em risco a saúde mental dos bombeiros ao longo de suas carreiras. Os participantes são avaliados na primeira semana do curso básico de formação e reavaliados periodicamente após o início das atividades profissionais. Há semelhanças entre as propostas do PSOBM e do ESB, mas o segundo avança as possiblidades de compreensão da dinâmica saúde mental-trabalho na medida em que cria uma avaliação prévia ao início da exposição a estressores operacionais e organizacionais e aprofunda a investigação sobre tais estressores no dia a dia de trabalho. 
A complementaridade entre os programas tem sido útil para informar as políticas de saúde no CBMMG. É possível que a experiência acumulada na instituição ao longo dos últimos anos contribua para o avanço das ações de Visat entre Corpos de Bombeiros, em especial no que tange a saúde mental. Nesse sentido, o objetivo do presente artigo foi descrever aspectos conceituais e metodológicos que subjazem o PSOBM e o ESB. Pretende-se ainda elencar desafios e possiblidades de desenvolvimento de ambos os projetos.

\section{VIGILÂNCIA EM SAÚDE MENTAL DOS BOMBEIROS DE MINAS GERAIS}

\subsection{Programa de Saúde Ocupacional Bombeiro Militar (PSOBM)}

O PSOBM foi idealizado por uma comissão de oficiais e praças de saúde pertencentes aos quadros do CBMMG. A comissão finalizou o projeto em outubro de 2015 com a publicação da Resolução N ${ }^{\circ} 640$ (MINAS GERAIS, 2015). Em 2016, foi realizada uma edição piloto do programa cujo principal objetivo foi testar sua viabilidade e identificar barreiras e facilitadores para a implementação no estado de Minas Gerais. Foram selecionadas duas unidades da região metropolitana de Belo Horizonte, denominadas Núcleos de Atenção Integral à Saúde (NAIS). Os NAIS são estruturas próprias do CBMMG onde atuam profissionais de saúde militares e, em alguns casos, civis contratados (LIMA, FROIS, BATISTA, 2019). Após a versão piloto, a partir de 2017, o programa passou a ser gradativamente expandido para unidades localizadas no interior do estado. Uma observação: o sistema de saúde do CBMMG é integrado ainda pela Polícia Militar de Minas Gerais (PMMG) e pelo Instituto de Previdência dos Servidores Militares de Minas Gerais (IPSM). Juntas, estas três instituições compõem o Sistema de Saúde dos militares estaduais (SiSau). Ao ser expandido para o interior, o PSOBM passou a ser realizado também nos NAIS da PMMG, exigindo ajustes e adaptações que ainda estão em 
Revista Científica do Corpo de Bombeiros Militar de Pernambuco

Artigo Publicado no Vol.06 N.16 - Edição Especial 2020 - ISSN 2359-4829

Versão on-line disponível em: http://www.revistaflammae.com

andamento. A proposta é contemplar os cerca de 5.700 bombeiros que atuam em 76 municípios de Minas Gerais.

A cada ano metade do efetivo das unidades é convocada em seu NAIS de referência para uma avaliação de saúde. Ou seja, a ideia é que cada bombeiro seja avaliado bienalmente. A Figura 1 apresenta o fluxo das etapas do PSOBM realizadas até o momento.

Figura 1 - Fluxo das etapas e público atendido nas avaliações clínicas periódicas no CBMMG (Período: 2016-2020). Programa de Saúde Ocupacional Bombeiro Militar (PSOBM)

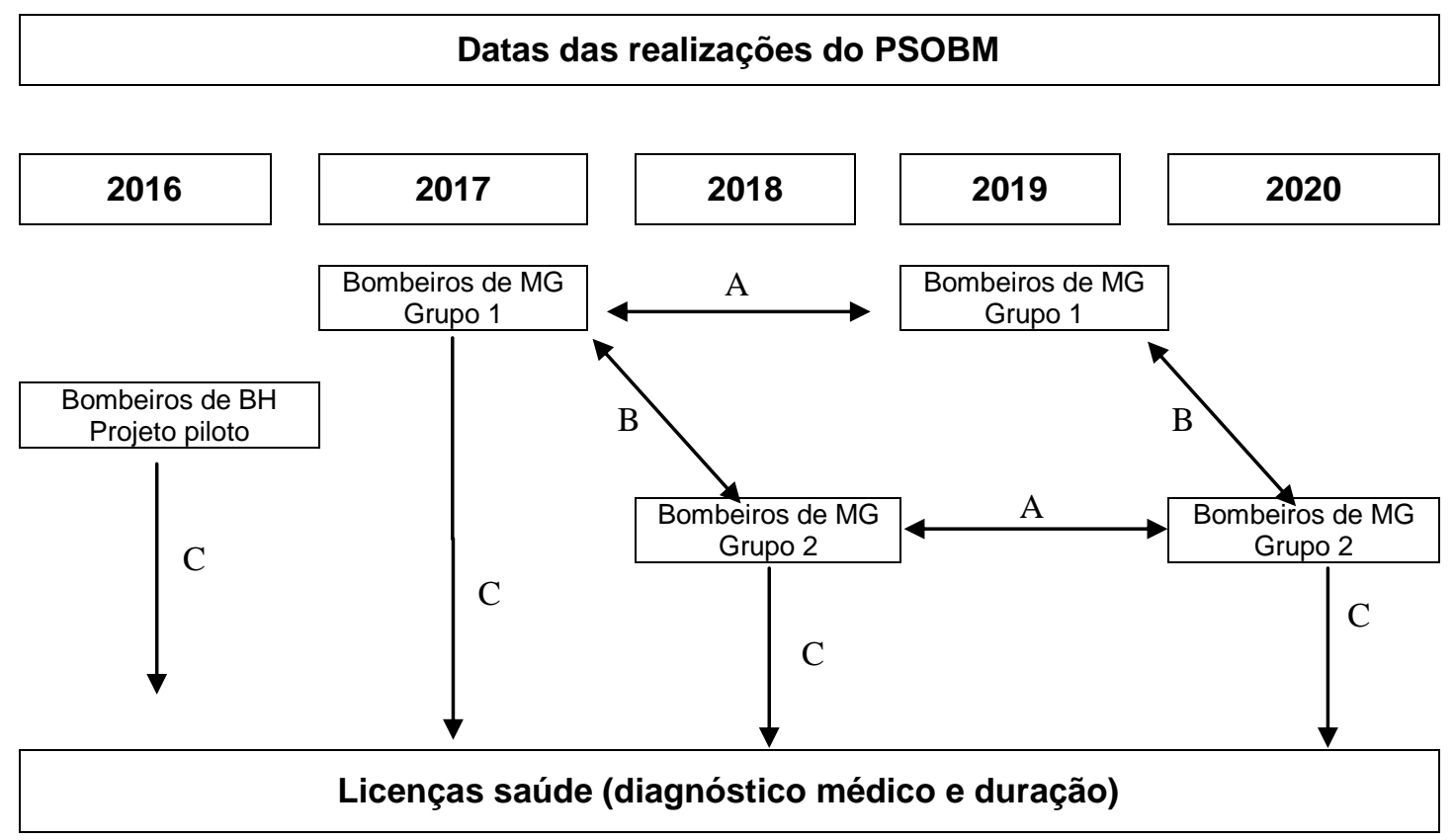

Nota: BH: Belo Horizonte; MG: Minas Gerais; Grupo 1: refere-se a metade do efetivo do CBMMG avaliado no PSO BM em 2017 e 2019; Grupo 2: refere-se a metade do efetivo do CBMMG avaliado no PSO BM em 2018 e 2020; ${ }^{A, B}$ : Comparação dos dois grupos de bombeiros militares nas avalições realizadas em diferentes momentos ${ }^{\mathrm{C}}$ : Comparação dos resultados das avaliações clínicas com as tendências de licenças saúde

Profissionais de diferentes especialidades atuam no desenvolvimento do programa, incluindo oficiais médicos, psicólogos e dentistas e praças auxiliares, como técnicos de enfermagem e de saúde bucal. As avaliações médica e odontológica são feitas em paralelo, de forma independente. Em 
ambos os casos, os oficiais de saúde realizam anamnese e exame clínico e solicitam exames complementares a depender do paciente (sexo, idade, histórico de saúde e função exercida na instituição). Ao final, os oficiais médico e dentista fazem orientações sobre saúde, indicam tratamentos, renovação de vacinas e emitem um parecer final sobre as condições de trabalho do bombeiro militar. Caso sua condição de saúde o impeça de realizar atividades profissionais, o médico ou o dentista recomendam afastamento total (licença) ou parcial (dispensa) do trabalho por um período determinado de tempo.

A avaliação psicológica é feita em duas etapas. Na primeira, os bombeiros convocados para o PSOBM preenchem um questionário de triagem sobre exposição a estressores operacionais (eventos traumáticos no trabalho) e sintomas relacionado à saúde mental. Tanto o questionário sobre exposição quanto os questionários para sintomas psíquicos foram traduzidos, adaptados e validados para a população brasileira (LIMA, VASCONCELOS, BARRETO, ASSUNÇÃO, 2016; LIMA, BARRETO E ASSUNÇÃO, 2012; MARI, WILLIAMS, 1986).

Na sequência, os bombeiros que apresentarem sintomas prováveis de adoecimento psíquico, de acordo com as normas dos questionários, são encaminhados para uma entrevista clínica com um oficial psicólogo. Durante a entrevista, o oficial faz orientações sobre saúde mental (psicoeducação), pode indicar a realização de um psicodiagnóstico completo ou tratamento psicoterápico e emite um parecer final sobre as condições de trabalho do avaliado. De forma semelhante à avaliação médica e odontológica, o psicólogo pode recomendar licença ou dispensa de saúde, caso necessário. 
Figura 2 - Informações solicitadas no protocolo de rastreio de sintomas psíquicos e exposição a estressores operacionais no CBMMG. Programa de Saúde Ocupacional do CBMMG (PSOBM)

\begin{tabular}{l} 
Variáveis \\
\hline 1. Informações sociodemográficas (idade, sexo, estado civil, escolaridade) \\
2. Características do trabalho no CBMMG (unidade, carga de trabalho, \\
atividades executadas mais frequentes) \\
3. Sintomas de ansiedade, depressão e estresse pós-traumático \\
4. Estressores ocupacionais (eventos que envolveram morte ou risco de morte \\
de pessoas)
\end{tabular}

\subsection{Estudo da Saúde do Bombeiro (ESB)}

O ESB foi idealizado em 2013, em parceria com o Laboratório de Avaliação e Intervenção na Saúde da Universidade Federal de Minas Gerais (UFMG). Sintomas de TEPT, ansiedade e depressão foram considerados desfechos centrais do projeto. As variáveis de exposição foram delimitadas de acordo com o modelo teórico-conceitual que fundamentou o desenvolvimento do ESB. Para os estressores operacionais, foi utilizado o conceito de evento traumático convergente ao adotado pela Associação de Psiquiatria Americana para o diagnóstico de TEPT (APA, 2013). Para os estressores organizacionais, foi adotado o Modelo Demanda-Controle (KARASEK, THOERELL, 1990).

O desenvolvimento do referencial teórico e das hipóteses resultou em dois protocolos de coleta de dados (Figura 3). O primeiro referente à primeira avaliação dos soldados durante o treinamento básico (avaliação inicial) e 0 segundo referente às avaliações subsequentes (reavaliações) ao longo da carreira. A estrutura dos protocolos da avaliação inicial e das reavaliações foram objeto do "Manual Explicativo do protocolo do ESB" (VASCONCELOS, OCHIAI, LIMA, NASCIMENTO, 2017), registrado na biblioteca da UFMG. Os protocolos foram compostos por instrumentos previamente validados para 0 contexto brasileiro e utilizados em pesquisas anteriores. 
Revista Científica do Corpo de Bombeiros Militar de Pernambuco

Artigo Publicado no Vol.06 N.16 - Edição Especial 2020 - ISSN 2359-4829

Versão on-line disponível em: http://www.revistaflammae.com

Figura 3 - Informações solicitadas na avaliação e reavaliações de bombeiros militares do CBMMG. Estudo da Saúde do Bombeiro (ESB).

\begin{tabular}{|c|c|}
\hline $\begin{array}{c}\text { Protocolos } \\
\text { ESB }\end{array}$ & Variáveis \\
\hline $\begin{array}{c}\text { Avaliação } \\
\text { inicial }\end{array}$ & $\begin{array}{l}\text { 1. Informações sociodemográficas (idade, sexo, estado civil, } \\
\text { escolaridade) } \\
\text { 2. Características ocupacionais prévias à admissão no CBMMG } \\
\text { (ocupação, tempo na ocupação) } \\
\text { 3. Status socioeconômico (renda familiar média, educação da mãe) } \\
\text { 4. Saúde geral (histórico de diagnóstico prévio de doenças crônicas, } \\
\text { uso de medicamentos psiquiátricos, peso e altura) } \\
\text { 5. Sintomas de ansiedade, depressão e estresse pós-traumático } \\
\text { 6. Funcionamento psicológico (crenças cognitivas, afeto e } \\
\text { personalidade) } \\
\text { 7. Exposição a eventos estressantes e traumáticos }\end{array}$ \\
\hline Reavaliações & $\begin{array}{l}\text { 1. Informações sociodemográficas (idade, sexo, estado civil, } \\
\text { escolaridade) } \\
\text { 2. Características do trabalho no CBMMG (unidade, carga de trabalho, } \\
\text { atividades executadas mais frequentemente) } \\
\text { 3. Saúdegeral (histórico de diagnóstico prévio de doencas crônicas, } \\
\text { uso de medicamentospsiquiátricos, uso de álcool e tabagismo) } \\
\text { 3. Sintomas de ansiedade, depressão e estressepós-traumático } \\
\text { 5. Funcionamento psicológico (crenças cognitivas, coping frente a } \\
\text { eventos traumáticos) } \\
\text { 6. Exposição a eventos estressantes } \\
\text { 7.Estressores ocupacionais (operacionais e organizacionais) }\end{array}$ \\
\hline
\end{tabular}

O desenho do projeto foi definido como longitudinal, prospectivo. A população alvo foi composta por uma coorte aberta, incluindo os soldados que ingressaram no CBMMG a partir de 2014. Além do requisito de iniciar a carreira na graduação de soldado, ter realizado o treinamento inicial na Academia de Bombeiros ( $A B M)$, em Belo Horizonte, foi um critério de inclusão no ESB. Considerou-se como critérios de exclusão a ausência no treinamento na data da coleta de dados (por falta ou licença) e a recusa em participar do projeto.

Ainda em 2013, um projeto piloto foi realizado para evitar possíveis vieses na coleta de dados. Buscou-se melhorar a compreensão dos itens, organiza-los em blocos homogêneos, e adequar itens específicos ao contexto de trabalho dos bombeiros militares. Os resultados foram satisfatórios, uma vez que o instrumento de coleta se mostrou viável e de fácil compreensão. Por fim, 
o projeto foi aprovado pelo CBMMG e pelo Comitê de Ética em Pesquisa da UFMG (CAAE: 15169813.1.0000.5149).

Em 2014, iniciaram-se as avaliações. Duas modalidades de coleta de dados têm sido adotadas deste então: face a face e virtual. A realização da avaliação inicial é face a face, centralizada na ABM, em salas de aula, durante o horário do treinamento. Após uma discussão sobre a relação entre saúde e trabalho, os bombeiros recém-admitidos são convidados a preencher 0 protocolo. Para cada reavaliação, os psicólogos responsáveis pelo projeto e uma equipe de alunos de graduação e pós-graduação do Departamento de Psicologia da UFMG percorrem as unidades do CBMMG onde os participantes estão alocados, realizam palestras sobre saúde mental e convidam os participantes a uma nova avaliação. Bombeiros pertencentes a unidades com menos de dez potenciais participantes do ESB recebem os convites por correio eletrônico e contato telefônico. A estratégia justifica-se pela complexidade logística e os custos envolvidos na realização de entrevistas presenciais durante as reavaliações. Na Figura 4, é possível observar o fluxo das etapas do ESB conduzidas até o momento. 
Revista Científica do Corpo de Bombeiros Militar de Pernambuco

Artigo Publicado no Vol.06 N.16 - Edição Especial 2020 - ISSN 2359-4829

Versão on-line disponível em: http://www.revistaflammae.com

Figura 4 - Fluxo das etapas e público atendido nas avaliações psicológicas periódicas dos soldados das turmas de 2014 e 2017 CBMMG (Período: 2014-2020).

Estudo da Saúde do Bombeiro (ESB)
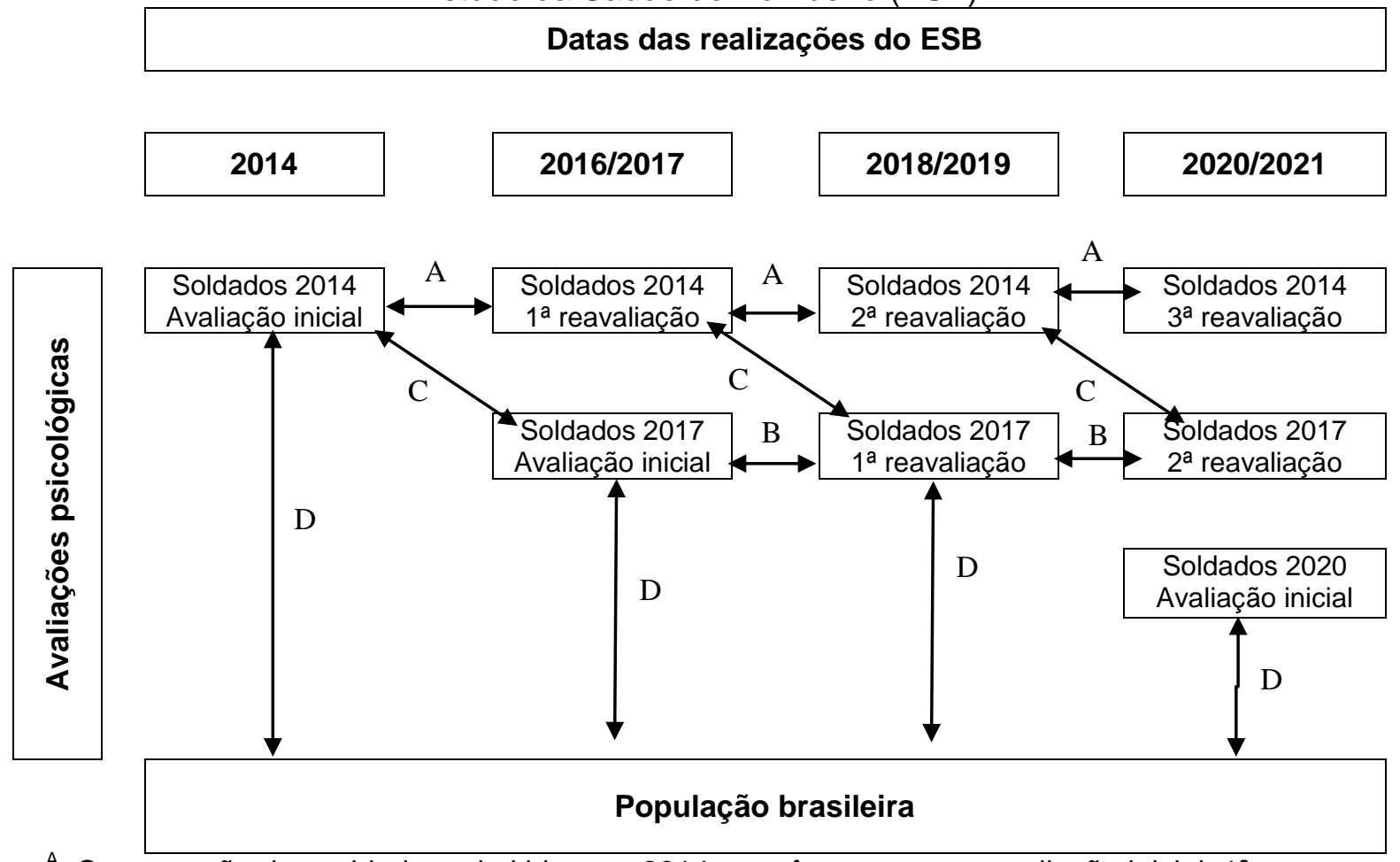

A: Comparação dos soldados admitidos em 2014 em três momentos: avaliação inicial, $1^{\text {a }}$ reavaliação, $2^{\mathrm{a}}$ reavaliação; ${ }^{\mathrm{B}}$ : Comparação dos soldados admitidos em 2017 em dois momentos: avaliação inicial, 1 a reavaliação; ${ }^{C}$ Comparação dos soldados admitidos em 2014 , 2017 e 2020: avaliações iniciais; ${ }^{D}$ Comparação dos soldados admitidos em 2014, 2017 e 2020 e a população brasileira com idade entre 18 a 30 anos: avaliações iniciais e prevalência de diagnósticos psiquiátricos conforme dados da Pesquisa Nacional de Saúde (PNS, BRASIL, 2013).

Os participantes têm como benefício o acesso a um relatório individual após a avaliação e cada reavaliação, com enfoque em eventuais sintomas de saúde mental e em comportamentos nocivos autorrelatados. Quando necessário, os bombeiros são encaminhados a profissionais de saúde para assistência médica ou psicológica. O procedimento foi adotado tanto em Belo Horizonte, quanto nos diversos municípios do interior de Minas Gerais. Até o momento, 1.094 bombeiros participaram do projeto.

A partir da avaliação inicial, os participantes passam a receber mensagens da equipe do ESB em datas comemorativas e informações sobre 
saúde mental de bombeiros. Espera-se ainda que se sintam motivados pela oportunidade de contribuir para a geração de conhecimento sobre a saúde dos bombeiros ao longo dos anos de trabalho. Esse aspecto tem sido confirmado pelas declarações espontâneas dos participantes após o preenchimento do questionário.

\section{VIGILÂNCIA EM SAÚDE MENTAL DO BOMBEIRO: CONTRIBUIÇÕES E DESAFIOS}

Programas como o PSOBM e o ESB foram estruturados de forma a reconhecer que os bombeiros são uma população exposta a estressores operacionais e estressores organizacionais específicos. É importante notar que, no cotidiano de funcionamento dos Corpos de Bombeiros, ambos os grupos de estressores podem variar em função das tarefas (resgate, atendimento pré-hospitalar, vistoria e prevenção) e do tipo de ocorrências atendidas (por exemplo, o rompimento da barragem em Brumadinho em 2019 ou o combate à pandemia de COVID-19 em 2020).

Os dois programas se propõem a avaliar e monitorar o desenvolvimento de sintomas de saúde mental entre os bombeiros de Minas Gerais (vigilância ativa). Os passos seguintes incluem organizar tais dados na forma de indicadores, discuti-los com trabalhadores e gestores e, por fim, estruturar ações de intervenção e políticas de saúde com base em um diagnóstico amplo (MAIZLISH, 2000). Este é o foco institucional do PSOBM e do ESB.

Os resultados obtidos têm trazido benefícios para a gestão em saúde e tomada de decisão do Comando do CBMMG. É importante notar que o PSOBM e o ESB comportam uma abordagem de intervenção primária (preventiva), que consiste em oferecer periodicamente informações sobre a saúde, permitindo identificar padrões, tendências e fatores de risco; mas também, uma abordagem de intervenção secundária e terciária, que consiste na orientação, tratamento e reabilitação de bombeiros militares que apresentam sinais de adoecimento (LIMA, ASSUNÇÃO, 2013). Nesse sentido, permitem diminuir a 
chance de surgimento e agravamento de doenças, o que impacta na redução dos dias de trabalho perdidos, redução dos custos com tratamento de saúde e geração de informações para a tomada de decisões. Em relação a este ponto, por exemplo, a experiência com ações em Visat concorreram para 0 desenvolvimento de um sistema de monitoramento das licenças médicas resultantes de casos suspeitos e confirmados da COVID-19. As informações sobre o número e duração dos afastamentos do trabalho têm sido úteis para respaldar ações de saúde visando a adoção de medidas de mitigação no combate à pandemia e decisões sobre alocação de recursos humanos e logísticos (MINAS GERAIS, 2020).

Além desta medida em um contexto de pandemia, nos últimos cinco anos, o PSOBM e o ESB permitiram o desenvolvimento de medidas institucionais especificamente voltadas para a saúde mental. Destaca-se o protocolo clínico para acompanhamento dos bombeiros militares que atuaram na ocorrência do rompimento da barragem de rejeitos da Vale, na cidade de Brumadinho. Tal protocolo ofereceu diretrizes aos profissionais de saúde da instituição ao contemplar estratégias de identificação daqueles com sintomas relacionados à saúde mental e estratégias de intervenção e tratamento (MINAS GERAIS, 2019).

Do ponto de vista individual, todo bombeiro militar atendido no PSOBM e ESB recebe orientações sobre sua própria saúde, com ênfase na descrição de sintomas e hábitos de vida. Quando necessário, o participante recebe orientações sobre benefícios potenciais da busca por atenção médica e psicológica. Atuar ativamente na prevenção e no alívio de sintomas de saúde mental contribui para minimizar o sofrimento e os prejuízos que podem acarretar na vida pessoal e profissional (TSUCHIYA et al., 2012; GLINA, ROCHA, 2010).

Além disso, um posicionamento institucional formalizado em programas voltados para a saúde mental dos bombeiros também contribui para a diminuição do estigma em relação aos transtornos mentais e para o 
fortalecimento do vínculo de confiança com psicólogos e psiquiatras (REGEHR, BOBER, 2005). Ao se reconhecer a importância da saúde mental e sua relação dinâmica com os estressores ocupacionais, se permite que estes temas sejam percebidos pelos bombeiros militares como inerentes à atividade que exercem. Em outras palavras, os programas favorecem o reconhecimento e o cuidado com a dimensão psíquica da saúde de todos.

Há ainda uma contribuição à população atendida pelos bombeiros militares. Problemas de saúde mental comprometem a capacidade de trabalho dos indivíduos em geral, independente da área em que atuam (TSUCHIYA et al., 2012). No caso dos bombeiros, a promoção da saúde e a prevenção de doenças permite um atendimento adequado em situações de urgência e emergência, em que se exige prontidão de resposta (LIMA, ASSUNÇÃO, 2011). Os programas citados contribuem para que a população possa contar com profissionais em condições físicas, cognitivas e emocionais satisfatórias.

Por fim, é importante elencar a contribuição para a literatura sobre Saúde do Trabalhador, em especial àquela voltada aos bombeiros brasileiros. Está reconhecido o efeito deletério à saúde diante da exposição a eventos estressantes e traumáticos no trabalho (VAN DER VELDEN, KLEBER, GRIEVINK, YZERMANS, 2010; LIMA, ASSUNÇÃO, 2011; LIMA, BARRETO, ASSUNÇÃO, 2015). Entretanto, projetos delineados para acompanhar a saúde psíquica destes profissionais ao longo do tempo, desde o início da carreira, são raros na literatura internacional. No Brasil, não há registro de estudos deste tipo. Portanto, os dois programas apresentados oferecem informações que podem contribuir para o preenchimento de lacunas sobre o que se sabe sobre a saúde de trabalhadores do setor de emergências em geral, bombeiros militares em particular. Ademais, o ESB busca reunir evidências que permitam o desenvolvimento de um modelo teórico-ocupacional sobre as interrelações entre os sintomas de transtornos mentais e aspectos ocupacionais no trabalho dos bombeiros. 
O desenvolvimento de ambos os projetos guarda desafios. O primeiro a ser ressaltado é logístico. A proposta do PSOBM é realizar avaliações de saúde mental bienalmente. Sua execução requer uma estrutura física para alcançar todas as unidades do estado, uma equipe de profissionais de saúde diversa e bem treinada e sistemas informatizados de registro e controle das avaliações. Conforme destacado, a ampliação do PSOBM para as cidades do interior de Minas Gerais exigiu o apoio da PMMG e adaptações nos fluxos e processos. No computo final, os resultados têm sido positivos, mas ainda há um caminho a ser percorrido. $O$ investimento em treinamento da equipe de saúde deve ser contínuo e incluir a rede de profissionais credenciados nas cidades onde não há médicos e psicólogos vinculados ao CBMMG e à PMMG. $O$ treinamento continuado da equipe responsável pelo ESB é um cuidado que vem sendo observado desde 2014, mas há limites para o deslocamento da equipe pelo estado. Portanto, o desafio logístico principal para este programa diz respeito aos custos com viagens e com softwares profissionais de coleta de dados virtuais.

Desafios metodológicos também estão presentes. No caso do ESB, após a avaliação inicial dos bombeiros militares, as demais reavaliações exigem que mensagens e informativos sobre o programa cheguem até eles de forma oportuna. Um dos grandes desafios de acompanhamentos longitudinais é a manutenção do vínculo entre os proponentes do projeto e os participantes (AQUINO et al., 2013). Por isso, é preciso planejar e monitorar estratégias que garantam adesão, minimizando perdas ao longo do tempo. A equipe responsável pelo ESB tem encontrado resultados satisfatórios por meio dos canais formais (e-mail institucional e intranet) e informais (telefone, grupos de WhatsApp) nos últimos anos. Textos, vídeos, mensagens e felicitações nas datas comemorativas são algumas das estratégias para informar e, ao mesmo tempo, manter o vínculo com os participantes.

Um outro aspecto metodológico desafiador é o registro e a organização sistemática das informações obtidas ao longo do tempo (DUNCAN et al., 2013). 
A partir das experiências com o PSOBM e com o ESB, percebeu-se que, na medida do possível, o registro das informações deve ser realizado virtualmente. O uso de softwares é preferível ao registro em papel, pois dispensa revisão de protocolos e codificação de informações antes de serem digitalizadas. $O$ registro em papel aumenta o risco de erros perda de dados. Programas de acesso livre são uma opção, embora as ferramentas pagas ou feitas sob medida para a instituição ofereçam mais recursos.

O armazenamento seguro das informações é um desafio ético. Dados sobre saúde devem ser sigilosos, mantidos sob responsabilidade da equipe à frente dos programas. Garantir tal sigilo é essencial para evitar a exposição de dados sensíveis, manter uma relação de confiança com os participantes e garantir as orientações preconizadas pelos órgãos de regulação profissional.

Por fim, é preciso ressaltar dois desafios muitas vezes subestimados em ações de Visat: o planejamento e a implementação de intervenções. Por um lado, os dados do PSOBM e do ESB permitem orientação e indicação de tratamento para cada bombeiro militar; por outro, oferecem subsídios para mudanças no ambiente e na organização do trabalho para toda a instituição. Mas estas últimas não dependem apenas dos profissionais de saúde. $\mathrm{Na}$ verdade, dependem principalmente dos trabalhadores na ponta da linha e dos gestores da instituição. O desafio aqui é favorecer o acesso de todos os membros do CBMMG às informações geradas, permitindo uma discussão ampla sobre os problemas prioritários e as possibilidades de mudança. Esta etapa final de retroalimentação exige esforço e organização por parte da equipe de saúde. Em suma, construir caminhos institucionais para discussão dos problemas identificados, busca por soluções e implementação destas soluções é o grande desafio das ações de Visat (MAIZLISH, 2000). 


\section{CONSIDERAÇÕES FINAIS}

O peso dos transtornos mentais sobre o bem-estar de trabalhadores é uma preocupação crescente na área da Saúde do Trabalhador. Respostas coordenadas e eficazes do poder público ao crescimento de problemas de saúde mental na população geral e entre profissionais de setores específicos é cada vez mais urgente. Não obstante, ações de Visat em saúde mental ainda são incipientes no Brasil. O PSOBM e o ESB têm contribuído para preencher essa lacuna no âmbito do CBMMG, em acordo com o plano estratégico institucional.

Lidar com prevenção e promoção em saúde mental de trabalhadores é um processo complexo e exige atenção simultânea a dimensões individuais, coletivas, organizacionais e socioculturais. No caso dos bombeiros, a alta demanda de trabalho e o contato diário com a morte e a violência podem resultar em consequências danosas para a saúde mental. Sabe-se que, para além do sofrimento individual, os sintomas podem acarretar absenteísmo e prejuízos para o desempenho das tarefas. Ressalta-se que, para além da oferta de opções de tratamento clínicos para aliviar sintomas, é preciso investir em mudanças na organização e nas condições do trabalho.

\section{AGRADECIMENTOS}

Agradecemos à Fundação de Amparo à Pesquisa do Estado de Minas Gerais (FAPEMIG) pelo apoio financeiro recebido para o desenvolvimento do ESB. Agradecemos também ao Comando-Geral e à Assessoria de Assistência à Saúde do CBMMG pelo apoio logístico e operacional na execução de ambos os projetos aqui descritos. Por fim, agradecemos a todos bombeiros militares do CBMMG, em especial às turmas de CFSd 2014 e CFSd 2017 pela participação nas avaliações. 


\section{REFERENCIAL}

AMATO, Tatiana de Castro et al. Trabalho, gênero e saúde mental: uma pesquisa quantitativa e qualitativa entre bombeiros. Cadernos de Psicologia Social do Trabalho, v. 13, n. 1, p. 103-118, 2010.

\section{AMERICAN PSYCHIATRY ASSOCIATION. Diagnostic and Statistical} Manual of Mental Disorders (DSM 5th ed.). Washington, DC: Author, 2013.

AQUINO, Estela et al. Recrutamento de participantes no Estudo Longitudinal de Saúde do Adulto. Revista de Saúde Pública, v.47, suppl.2, p. 10-18, 2013

BERGER, William et al. Partial and full PTSD in Brazilian ambulance workers: prevalence and impact on health and on quality of life. Journal of Traumatic Stress, v. 20, n. 4, p. 637-642, 2007.

BRASIL. Ministério da Fazenda / Instituto Nacional do Seguro Social. Anuário Estatístico da Previdência Social. Disponível em http://www.previdencia.gov.brBrasília, DF: 2017.

BRASIL. Instituto Brasileiro de Geografia e Estatística (IBGE). Pesquisa Nacional de Saúde 2013. Percepção do estado de saúde, estilo de vida e doenças crônicas. Brasil, Grandes Regiões e Unidades da Federação. Rio de Janeiro: IBGE; 2014.

DUNCAN, Bruce Bartholow et al. Gerência de informação em estudos multicêntricos: o Estudo Longitudinal de Saúde do Adulto. Revista de Saúde Pública, v. 47, suppl.2, p.95-104, 2013.

FIORIN, Priscila Maria Marcheti. Absenteísmo no Corpo de Bombeiros Militar do município de Campo Grande, MS. 63f. Dissertação (Mestrado em Saúde e Desenvolvimento) - Programa de Pós-Graduação em Saúde e Desenvolvimento na Região Centro-Oeste, Universidade Federal de Mato Grosso do Sul, Mato Grosso do Sul, 2013.

GLINA, Débora Miriam Raab; ROCHA, Lys Esther. Saúde Mental no Trabalho - da teoria à prática. São Paulo: Roca, 2010.

GOETZEL, Ron et al. Mental Health in the workplace: a call to action proceedings from the mental health in the workplace: Public health summit. Journal of Occupational and Environmental Medicine, v. 60, n. 4, p. 322330, 2018. 
Revista Científica do Corpo de Bombeiros Militar de Pernambuco

Artigo Publicado no Vol.06 N.16 - Edição Especial 2020 - ISSN 2359-4829

Versão on-line disponível em: http://www.revistaflammae.com

KARASEK, Robert; THEORELL, Tores. Healthy work: stress, productivity, and the reconstruction of working life. US: Basic Books, 1990. 381p.

LEÃO, Luís Henrique da Costa; GOMEZ, Carlos Minayo. A questão da saúde mental na vigilância em saúde do trabalhador. Ciência \& Saúde Coletiva, v. 19, n. 12, p. 4649-4658, 2014

LIMA, Eduardo de Paula; ASSUNÇÃO, Ada Ávila. Prevalência e fatores associados ao Transtorno de Estresse Pós-Traumático (TEPT) em profissionais de emergência: uma revisão sistemática da literatura. Revista Brasileira de Epidemiologia, v. 14, n. 2, p. 217-30, 2011.

LIMA, Eduardo de Paula; BARRETO, Sandhi Maria; ASSUNÇÃO, Ada Ávila. Factor structure, internal consistency and reliability of the Posttraumatic Stress Disorder Checklist (PCL): an exploratory study. Trends in Psychiatry and Psychotherapy, v. 34, n. 4, p. 215-222, 2012.

LIMA, Eduardo de Paula; ASSUNÇÃO, Ada Ávila. Saúde mental e trabalho: evidências empíricas e estratégia de abordagem. In: ASSUNÇÃO Ada Ávila. Promoção da saúde dos servidores municipais: abordagem e estratégias de intervenção. 88p. 2013.

LIMA, Eduardo de Paula. Transtorno de estresse pós-traumático em bombeiros de Belo Horizonte. 183f. Tese (Doutorado em Saúde Pública) Faculdade de Medicina, Universidade Federal de Minas Gerais, Belo Horizonte, Minas Gerais, 2013.

LIMA, Eduardo de Paula; BARRETO, Sandhi Maria; ASSUNÇÃO, Ada Ávila. Prevalência de depressão em bombeiros. Cadernos de Saúde Pública, v. 31, n. 4, p. 733-743, 2015.

LIMA, Eduardo de Paula; VASCONCELOS, Alina Gomide; BARRETO, Sandhi Maria; ASSUNÇÃO Ada Ávila. Lista de eventos traumáticos ocupacionais para profissionais de emergências: adaptação e validação. Avaliação Psicológica, v. 15, n. 3, p. 391-401, 2016.

LIMA, Eduardo de Paula; FROIS, Luciana; BATISTA, Andreia Geraldo. Vigilância em saúde mental no Corpo de Bombeiros Militar de Minas Gerais (CBMMG): implementação do Programa de Saúde Ocupacional (PSOBM). Manuscrito apresentado no formato de apresentação oral em: XIX Seminário Nacional de Bombeiros, São Luis, Maranhão, 2019.

LOPES, Vanessa Rodrigues. O papel do suporte social no trabalho e na resiliência no aparecimento de Burnout - um estudo com bombeiros militares. 204f. Dissertação (Mestrado em Psicologia) - Departamento de 
Revista Científica do Corpo de Bombeiros Militar de Pernambuco

Artigo Publicado no Vol.06 N.16 - Edição Especial 2020 - ISSN 2359-4829

Versão on-line disponível em: http://www.revistaflammae.com

Psicologia, Universidade Federal de Uberlândia, Uberlândia, Minas Gerais, 2010.

MAIZLISH, Neil. Work place health surveillance: an action-oriented approach. New York: Oxford University Press, 2000.

MARI, Jair de Jesus; WILLIAMS, Paul. A validity study of a psychiatric screening questionnaire (SRQ-20) in primary care in the city of São Paulo. The British Journal of Psychiatry, v. 148, p. 23-26, 1986.

TSUCHIYA, Masao et al. Impact of mental disorders on work performance in a community sample of workers in Japan: The World Mental Health Japan Survey 2002-2005. Psychiatry Research, v.198, n. 1, p. 140-5, 2012.

MINAS GERAIS. Corpo de Bombeiros Militar de Minas Gerais. Estabelece o Programa de Saúde Ocupacional Bombeiro Militar (PSOBM) no CBMMG. Resolução n. 640, de 15 de outubro de 2015. Belo Horizonte, Minas Gerais, 2015.

MINAS GERAIS.Corpo de Bombeiros Militar de Minas Gerais. Plano de Comando 2015/2016. Belo Horizonte, Minas Gerais, 2017.

MINAS GERAIS. Polícia Militar de Minas Gerais e Corpo de Bombeiros Militar de Minas Gerais. Protocolo clínico para acompanhamento dos militares que participaram da ocorrência do rompimento da barragem em Brumadinho - MG. 22f. Publicado em 04 de fevereiro de 2019, Belo Horizonte, Minas Gerais, 2019.

MINAS GERAIS. Corpo de Bombeiros Militar de Minas Gerais. Boletim Epidemiológico da Assessoria de Assistência à Saúde: análise diária dos afastamentos do trabalho por motivo de saúde durante as ações de resposta do Corpo de Bombeiros Militar de Minas Gerais (CBMMG) à pandemia da COVID-19. Belo Horizonte, Minas Gerais, 2020.

MONTEIRO, Rodrigo Crispim; LIMA, Eduardo de Paula. Lista de eventos traumáticos em combate a incêndio urbano (LET-CIU): um estudo piloto.

Vigiles - Revista de Defesa Civil, Defesa Social e Segurança Pública, v. 1, p. 19-30, 2018.

PEREIRA, Gustavo Klauberg. Associação entre variáveis ocupacionais e prevalência em agravos à saúde em policiais e bombeiros militares de Santa Catarina. 88f. Dissertação (Mestrado em Psicologia) - Universidade Federal de Santa Catariana, Florianópolis, Santa Catariana, 2017. 
REGEHR, Cheryl\& BOBER, Ted. In the line of fire: trauma in the emergency services. US: Oxford University Press, 2005.

STEEL Zachary, et al. The global prevalence of common mental disorders: a systematic review and meta-analysis 1980-2013. International Journal of Epidemiology, v. 43, n. 2, p. 476-493, 2014.

TEOH, Kevin; LIMA, Eduardo de Paula; VASCONCELOS, Alina Gomide; NASCIMENTO, Elizabeth; COX, Tom. Trauma and work factors as predictors of firefighters psychiatric distress. Occupational Medicine-Oxford, v. 69, p. 1, 2019.

VAN DER VELDEN, Peter; KLEBER, Rolf; GRIEVINK, Linda; YZERMANS, Joris. Confrontations with aggression and mental health problems in police officers: the role of organizational stressors, life-events and previous mental health problems. Psychological Trauma Theory Research Practice and Policy, v. 2, n. 2, p. 135-144, 2010.

VASCONCELOS, Alina Gomide; OCHIAI, Maki; LIMA, Eduardo de Paula; NASCIMENTO, Elizabeth. Manual explicativo do protocolo do Estudo da Saúde do Bombeiro - ESB. Belo Horizonte: Laboratório de Avaliação das Diferenças Individuais /FAFICH- UFMG, 2017. 